\title{
Anaesthesiologists' simulation training during emergencies in obstetrics
}

\author{
Volodymyr V. Artyomenko, Volodymyr M. Nosenko
}

Odessa National Medical University, Odessa, Ukraine, Department of Simulation Medicine, Odessa, Ukraine, Educational-innovative Center for Physician Practical Training

\begin{abstract}
Background and aims: Methods of simulation training and quality assessment during obstetric emergencies are still ambiguous. The aim of this study was to evaluate the effectiveness of anaesthesiologists' simulation training for emergency situations in obstetrics.

Methods: We conducted a prospective, descriptive, and comparative study to evaluate the anaesthesiologists' simulation training effectiveness during obstetrical emergencies. Data of 109 obstetrical anaesthesiologists trained over two years for invasive procedures and cardiopulmonary resuscitation, highfidelity scenarios and medical personnel teamwork included were analyzed. We used the two-sided t-test $(\mathrm{p}<0.05$ considered significant).

Results: We noted during the fifth training sessions, the anaesthesiologists had a significant manipulation time decrease for all skills compared to the ones assessed during their first training session $(p<0.01)$. The 100 -grade scale scores for all invasive techniques significantly improved during the anaesthesiologists' training $(\mathrm{p}<0.01)$. Cardiopulmonary resuscitation effectiveness and team work also improved significantly during the fifth session $(\mathrm{p}<0.01)$.

Conclusions: As a result of simulation training, significant improvement of speed and quality indicators, for invasive techniques in obstetrical emergency states treatment, was noted. For the fifth training sessions, there was a decrease in the practical skills execution time. The overall effectiveness and teamwork quality for cardiopulmonary resuscitation showed significant improvement.
\end{abstract}

Keywords: simulation medicine, simulation training, emergency states, obstetrics, anaesthesiology

Received: 20 December 2016 / Accepted: 6 March 2017

Rom J Anaesth Int Care 2017; 24: 37-40

\section{Introduction}

Anaesthesiologists who work in obstetrics often face urgent conditions which need appropriate modern standards of care. Methods of simulation training application and quality assessment, during obstetrical emergencies, are still ambiguous, many questions remaining open regarding the frequency of training, the teaching methodology - especially for complicated invasive procedures [1], the scenario simulation model

Address for correspondence:

Volodymyr Viktorovich Artyomenko Odessa National Medical University Department of Simulation Medicine Valihovsky lane, 2, Odessa, 65082, Ukraine

E-mail: vartyomenko2008@yandex.ru
[2], and the parameters to be evaluated [3]. Doctors have to be good team players and their training must systematically include teamwork skills. Teamworkrelated competencies are relatively new considerations in the arena of health care [4].

The aim of this study was to evaluate the effectiveness of anaesthesiologists' simulation training for urgent conditions management in obstetrics.

\section{Methods}

Data of 109 obstetric anaesthesiologists, trained in our center during two years (2014-2016), were included. Anaesthesiologists were assessed during several simulation training sessions.

We used Simulab company (S402 Arterial and Venous Patient Training Arm, FemoraLineMan, 
TraumaMan, CentralLineMan, Ultrasound Thoracentesis Model THM-20) and Gaumard company (Noelle S575.100 for high-fidelity-simulation and Super Chloe Patient Care Simulator) simulators and practical skills trainers.

During classes, conditions for independent performance of diagnostic and therapeutic manipulations under the guidance of medical and teaching staff, were created sequentially. Teaching was performed in small groups, training on models, phantoms, dummies, and high-fidelity robot simulators. There were at least five daily training sessions in emergency conditions settings. We used several simulation scenarios of emergency and critical states in pregnant women, in accordance with Unified clinical protocols of emergency medical care (Ministry of Health, Ukraine, 2014-2016). These scenarios were «cardiac arrest», «respiratory failure», «hemorrhagic shock», «severe pre-eclampsia» and «eclampsia», «hydrothorax», «thromboembolism», «amniotic fluid embolism». We used real-time ultrasound guidance during central venous catheterization, thoracocentesis, and others. Clinical situations were studied using multiple scenarios for high-fidelity robot simulators with realistic feedback and flexible programming regarding obstetric emergency conditions. Doctors were trained for skills of individual and team cardiopulmonary resuscitation and artificial ventilation, laryngeal mask insertion, oro-tracheal intubation, and coniotomy performance. Team training sessions with several anaesthesiologists, nurses and midwives to act in a particular clinical situation, to optimize teamwork, to improve collaboration, and to learn allocate responsibilities clearly in the team, were carried out.

During debriefing, the teaching instructor together with a psychologist conducted a detailed analysis of operations and developed a single algorithm $[5,6]$. The assessment of practical skills for treating emergency conditions, before and after the simulation training, was performed. We evaluated the correct selection of diagnostic and treatment algorithms, manual skills, and theoretical knowledge, as well as tools and skills to use these. Among practical skills we included: catheterization of peripheral veins, internal jugular, subclavian and femoral vein puncture, pleural cavity puncture, laryngeal mask insertion, oro-tracheal intubation, coniotomy, basic cardiopulmonary resuscitation skills (time to register the effectiveness of the cardiopulmonary resuscitation and overall assessment of individual and team performance). For evaluation, we used a 100 - points scoring system (during analysis, the results were standardized by transforming the raw results into a 100-grade scale (0-100 points)) [7]. Statistical analysis was carried out using Microsoft Excel 2007. We processed data obtained during several training sessions. The two-sided t-test was used to compare results between the different training sessions, $(p<$ 0.05 was considered significant). Continuous variables were expressed as the mean \pm standard deviation.

\section{Results}

We included 109 anaesthesiologists (63 male and 46 female), with a mean (SD) age of 32.3 (3.1) years and a mean (SD) work experience of 7.2 (2.3) years. We studied the duration of manipulation (taking into account all the time of antiseptics, anaesthesia, etc.) in dynamics, during the actual training or video (Table 1).

During the fifth training sessions, in comparison with the first training sessions, the anaesthesiologists had significant manipulation time decrease for all skills ( $p$ $<0.01$ ) (Figure 1).

The 100-grade scale scores for all invasive techniques significantly improved during the anaesthesiologists' training $(\mathrm{p}<0.01)$ (Table 2). Cardiopulmonary resuscitation effectiveness and team work comparing the first and fifth training sessions also improved significantly $(\mathrm{p}<0.01)$.

Table 1. Practical skills dynamics duration in the obstetrical emergency conditions treatment $\mathrm{n}=109$ (results are presented as mean \pm standard deviation)

\begin{tabular}{lcc}
\hline Practical skill type & $\begin{array}{c}\text { Manipulation duration during } \\
\text { the first training (seconds) }\end{array}$ & $\begin{array}{c}\text { Manipulation duration during } \\
\text { the fifth training (seconds) }\end{array}$ \\
\hline Peripheral veins catheterization & $18.03 \pm 1.97$ & $11.83 \pm 1.52^{*}$ \\
Internal jugular vein catheterization & $131.16 \pm 12.69$ & $64.05 \pm 6.90^{*}$ \\
Subclavian vein catheterization & $171.93 \pm 11.59$ & $115.98 \pm 7.61^{*}$ \\
Femoral vein catheterization & $157.86 \pm 22.40$ & $105.24 \pm 14.93^{*}$ \\
Pleural cavity puncture & $274.46 \pm 65.69$ & $158.40 \pm 35.00^{*}$ \\
Laryngeal mask insertion & $106.20 \pm 25.64$ & $64.14 \pm 8.79^{*}$ \\
Oro-tracheal intubation & $191.75 \pm 35.79$ & $88.37 \pm 10.38^{*}$ \\
Coniotomia & $106.00 \pm 10.38$ & $62.64 \pm 11.58^{*}$ \\
The effective cardiopulmonary resuscitation & $364.11 \pm 17.31$ & $124.11 \pm 17.31^{*}$ \\
\hline * statistically significant differences of the performance time during the fifth training session compared to the manipulation during \\
the first training $(\mathrm{p}<0.01)$
\end{tabular}




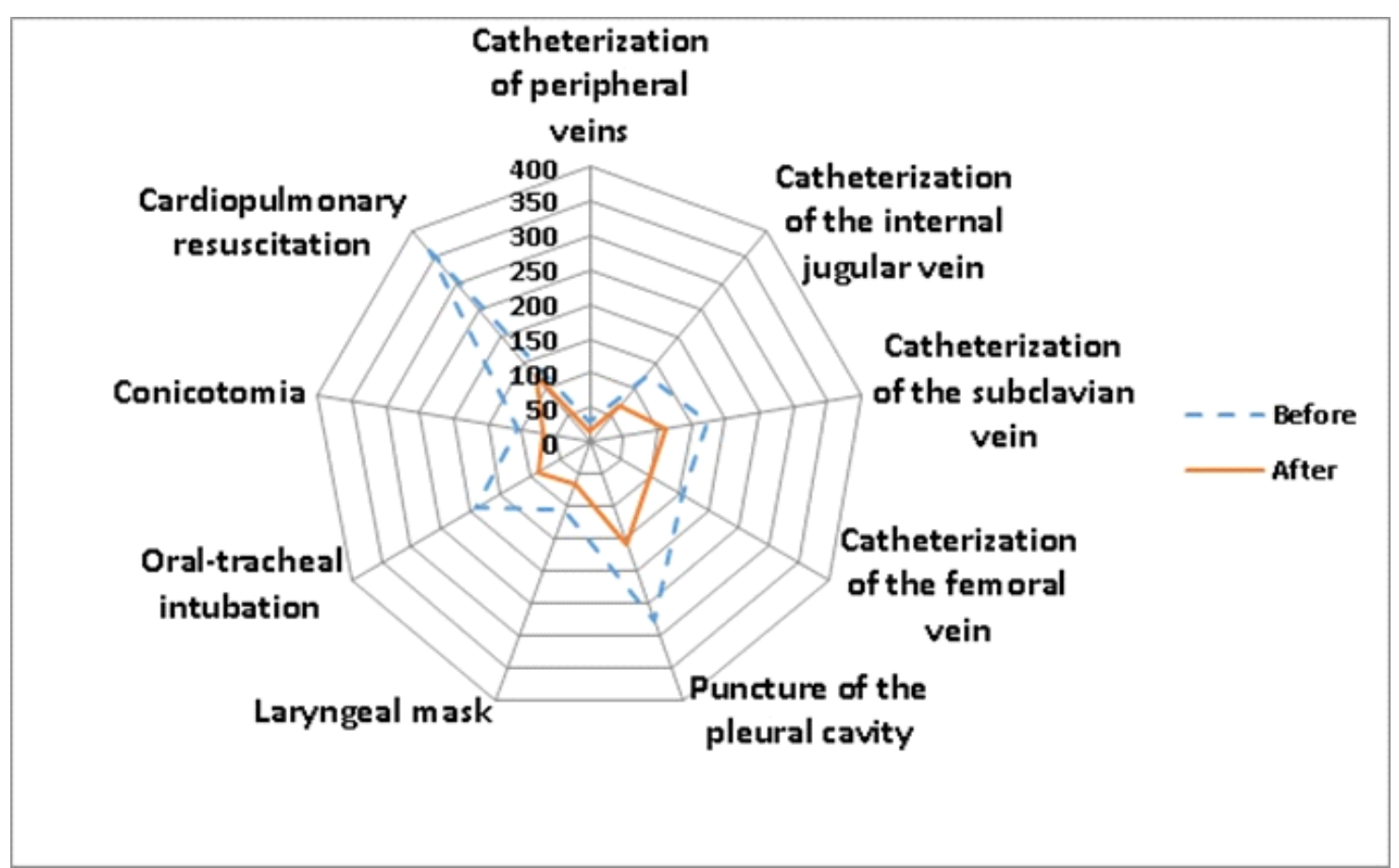

Fig. 1. Execution time of practical skills during first and fifth training for obstetrical emergencies, in seconds

Table 2. Skills performance scores (0-100 scale) during obstetrical emergency states (results are expressed as mean \pm standard deviation)

\begin{tabular}{lcc}
\hline Practical skills & During the first training & During the fifth training sessions \\
\hline Peripheral veins catheterization & $63.25 \pm 6.65$ & $87.64 \pm 1.50^{*}$ \\
Internal jugular vein catheterization & $58.80 \pm 6.89$ & $85.01 \pm 5.57^{*}$ \\
Subclavian vein catheterization & $58.81 \pm 6.91$ & $84.85 \pm 5.59^{*}$ \\
Femoral vein catheterization & $57.73 \pm 6.91$ & $84.91 \pm 5.66^{*}$ \\
Pleural cavity puncture & $58.61 \pm 6.22$ & $84.13 \pm 5.74^{*}$ \\
Laryngeal mask insertion & $57.84 \pm 6.80$ & $85.14 \pm 5.59^{*}$ \\
Oro-tracheal intubation & $58.71 \pm 6.57$ & $84.89 \pm 5.80^{*}$ \\
Coniotomia & $58.76 \pm 6.28$ & $84.69 \pm 5.55^{*}$ \\
The effective cardiopulmonary resuscitation & $58.71 \pm 6.53$ & $84.57 \pm 5.66^{*}$ \\
\hline
\end{tabular}

* statistically significant differences scores for the fifth training sessions compared to the manipulation during the first training $(\mathrm{p}<0.01)$

\section{Discussion}

Anaesthesiology specialists' high quality medical education is still problematic. Few studies have shown direct improvements in clinical outcomes from the use of simulation for training. [8]. One of the main problems is the lack of focus on learning algorithm actions in extreme situations, especially rapid decision making and seamless execution of manipulations in unusual circumstances in cooperation with experts in various fields [9]. Emergency conditions in obstetrics often require complex manipulations associated with possible risks for both mothers and babies. Some emergency situations may occur infrequently for each anaesthesiologist in practice [10]. The occurrence of urgent clinical situations, which are a real danger for the patient or for the newborn, require immediate treatment [11]. Thus, in this situation, as in no any other, the important role of simulation training arises [12].

Since 2014, the first Department of Simulation Medicine and Educational-Innovation Center for the physicians' practical training started functioning at the Odessa National Medical University, Ukraine [13]. One of the main directions of activity was to develop new and innovative approaches to medical education in the country. It is important to work out the theoretical and practical skills educational guidelines impose, and to gain experience for effective teamwork. As a result of research in this field, our own methods of both educational and practical aspects of anaesthesiologists training were implemented. More than 200 obstetrical 
anaesthesiologists have already undergone training in this center.

Implementation of simulation training represents a priority task for modern medical education due to a large gap between the traditional training and the requirements of modern medicine [8].

Simulation training of anaesthesia specialists for pregnant patients has been studied in a small number of research studies [8]. This is particularly important for obstetric anaesthesia specialists.

Previously, a project to improve emergency medical care in Latin America (SEMILLA) was developed, observing that the execution of practical skills and leadership skills in a team had improved by about 2.5 times [9]. We also observed approximately a 2-fold decrease in execution times in our study. The main common element, in the above cited project and our study, is the belief that the aim of simulation training is to improve health outcomes and survival of individual patients. One of the limitations of our study is that we did not evaluate the economic efficiency of simulation trainings. However, we set that purpose for the future. This includes reducing the time of temporary disability, the number of complications, mortality.

The first studies on the effectiveness of simulation training have already been published $[3,6,7,9,10]$. We took them as a basis and applied the system to our conditions.

Special attention was paid to team training simulation. Despite the large number of medical equipment, the human factor remains one of the most important to influence patients' outcomes. This is especially true in the work of anaesthesiologists and intensive care specialists, particularly in emergency conditions. One of the most important tasks of our work was to show the improved coherence and effectiveness of team work as a result of simulation training. It is important that professionals who work as a team in their practice (adult and paediatric anaesthesiologists, obstetrician, neonatologist, midwife) undergo joint simulation training.

\section{Conclusions}

1. As a result of simulation training, we found a significant improvement of speed indicators and the basic manipulations' scores in obstetrical emergency states treatment provided by anaesthesiologists.

2. During the fifth training, overall assessment and evaluation of the teamwork quality in cardiopulmonary resuscitation showed a significant improvement.

3. We recommend the use of different techniques of simulation education such as skills and teams training sessions in anaesthesiologists' modern medical education, especially in obstetrical emergency conditions, aiming to improve skills and promote patient safety.

\section{Conflict of interest}

Nothing to declare

\section{References}

1. Duncan JR, Henderson K, Street M, Richmond A, Klingensmith $\mathrm{M}$, Beta $\mathrm{E}$, et al. Creating and evaluating a data-driven curriculum for central venous catheter placement. J Grad Med Educ 2010; 2: 389-397. DOI: 10.4300/JGME-D-10-00007.1

2. Binstadt E, Donner S, Nelson J, Flottemesch T, Hegarty C. Simulator training improves fiber-optic intubation proficiency among emergency medicine residents. Acad Emerg Med 2008; 15: 1211-1214. DOI: 10.1111/j.1553-2712.2008.00199.x

3. Devitt JH, Kurrek MM, Cohen MM, Cleave-Hogg D. The validity of performance assessments using simulation. Anesthesiology 2001; 95: 36-42

4. Lateef F. Simulation-based learning: Just like the real thing. J Emerg Trauma Shock 2010; 3: 348-352. DOI: 10.4103/09742700.70743

5. Nyström S, Dahlberg J, Edelbring S, Hult H, Dahlgren MA. Debriefing practices in interprofessional simulation with students: a sociomaterial perspective. BMC Med Educ 2016; 16: 148. DOI: $10.1186 / \mathrm{s} 12909-016-0666-5$

6. Artyomenko VV, Elchaninova SI, Semchenko SS, Yegorenko OS, Novikov DA, Nosenko VM. The role of psychological training in the simulation education. Humanitarian Bulletin SU "Pereyaslav-Khmelnitsky Pedagogical University by $\mathrm{H}$. Skovoroda" Gnosis 2015; 36(Suppl.1): 32-39

7. Korzeniewska-Eksterowicz A, Przysło Ł, Kędzierska B, Stolarska M, Młynarski W. The impact of pediatric palliative care education on medical students' knowledge and attitudes. Scientific World Journal 2013: 498082. DOI: 10.1155/2013/498082

8. Okuda Y, Bryson EO, DeMaria S Jr, Jacobson L, Quinones J, Shen B, et al. The utility of simulation in medical education: what is the evidence? Mt Sinai J Med 2009; 76: 330-343. DOI: $10.1002 / \mathrm{msj} .20127$

9. Dîrzu DS, Copotoiu SM. Effectiveness versus efficiency in a medical skills laboratory. Rom J Anaesth Int Care 2015; 22: 35-39

10. Norris EM, Lockey AS. Human factors in resuscitation teaching. Resuscitation 2012; 83: 423-427. DOI: 10.1016/ j.resuscitation.2011.11.001

11. Taira BR, Orue A, Stapleton E, Lovato L, Vangala S, Tinoco LS, et al. Impact of a novel, resource appropriate resuscitation curriculum on Nicaraguan resident physician's management of cardiac arrest. J Educ Eval Health Prof 2016; 13: 25. DOI: $10.3352 /$ jeehp. 2016

12. Napier F, Davies RP, Baldock C, Stevens H, Lockey AS, Bullock I, et al. Validation for a scoring system of the ALS cardiac arrest simulation test (CASTest). Resuscitation 2009; 80: 1034-1038. DOI: $10.1016 /$ j.resuscitation.2009.04.043

13. Establishing a Simulation Center: Essentials and Guidelines. Experience of the Mother and Child Health Project / Handbook. Kyiv, Ukraine: Vistka, 2015. http://www.ebcog.eu/doc/ Simulation_Handbook_lr.pdf 